\title{
Síndrome de Churg Strauss como diagnóstico diferencial de poliposis nasosinusal, a propósito de un caso
}

\section{Churg Strauss Syndrome as a differential diagnosis of nasosinusal poliposis, case report}

\author{
Cecilia Sedano $\mathbf{M}^{1}$, Carlos Morales A , Jorge Castillo A², Daniel Cantero C¹.
}

\begin{abstract}
RESUMEN
El síndrome de Churg-Strauss (SCS) es una vasculitis necrotizante sistémica de etiología desconocida, que afecta vasos sanguíneos de pequeño y mediano tamaño. Característicamente se definen tres estadios: prodrómico, hipereosinofílico y una etapa de vasculitis sistémica. Dentro del prodrómico se encuentran manifestaciones del área otorrinolaringológica, que suelen preceder por años a las manifestaciones propias de las vasculitis. Entre ellas destacan la otitis media crónica, rinitis alérgica y rinosinusitis crónica poliposa. Reportamos el caso de un paciente de sexo masculino de 64 años con poliposis nasosinusal bilateral, con biopsia compatible con SCS, encontrándose en estado prodrómico de la enfermedad. Además, presentamos una revisión de la literatura sobre esta patología.
\end{abstract}

Palabras clave: Síndrome de Churg Strauss, vasculitis, pólipos nasales.

\begin{abstract}
Churg-Strauss syndrome (CSS) is a necrotizing systemic vasculitis of unknown etiology, which involves small and medium-sized vessels. It is characterized by three stages: prodromic, hypereosinophilic and systemic vasculitis. In the prodromal stage we can find otolaryngological manifestations (including chronic otitis media, allergic rhinitis, chronic rhinosinusitis with polyps) that usually precede the proper manifestations of the vasculitis by years. Here, we report a 64-year old male patient with sinonasal polyps and biopsy compatible with CSS, being in the prodromal stage of the illness. Also, we present a review of this disease.
\end{abstract}

Key words: Churg Strauss Syndrome, vasculitis, nasal polyps.

1 Médico del Servicio de Otorrinolaringología, Hospital Barros Luco Trudeau.

2 Médico del Servicio de Anatomía Patológica, Hospital Barros Luco Trudeau.

Recibido el 14 de agosto, 2017. Aceptado el 23 de septiembre, 2017. 


\section{INTRODUCCIÓN}

El síndrome de Churg-Strauss (SCS) o granulomatosis alérgica corresponde a una vasculitis necrotizante sistémica de etiología desconocida que afecta vasos sanguíneos de pequeño y mediano calibre ${ }^{1}$. Fue descrito por primera vez en el año 1951 por Jacob Churg y Lotte Strauss ${ }^{2}$. Usualmente se presenta entre los 14 y 75 años, con un promedio de edad de 50 años ${ }^{3}$. Es una patología infrecuente, tiene una incidencia de 1,3 a 6,8 casos por millón de habitantes por año ${ }^{4}$. Desde 1990 el Colegio Americano de Reumatología incluyó la patología de senos paranasales como uno de los seis criterios para el diagnóstico de esta enfermedad ${ }^{5}$.

Aun cuando se considera como una vasculitis asociada a ANCA, sólo se encuentra positivo en $40 \%$ de los casos, de éstos, el $75 \%$ corresponde a un patrón pANCA, MPO positivo ${ }^{6}$. En su evolución se distinguen tres estadios: prodrómico, eosinofílico (hiperreosinofilia $>10 \% \quad 0>1.500 / \mathrm{mm}^{3}$ ) y la etapa de vasculitis sistémica con granulomas eosinofílicos extravasculares ${ }^{4}$. Las manifestaciones otorrinolaringológicas se encuentran principalmente en el periodo prodrómico; presentándose como rinitis alérgica, rinosinusitis recurrente, rinosinusitis crónica poliposa, entre otros ${ }^{7}$. Los pólipos son, a simple vista, indistinguibles de los pólipos inflamatorios de la rinosinusistis crónica habitual y se presentan en el $25 \%$ a $50 \%$ de los casos ${ }^{4}$. Además de las manifestaciones nasosinusales, el SCS puede inicialmente manifestarse con otitis media crónica, parálisis facial o patología del oído interno, manifestada por hipoacusia sensorioneural generalmente bilateral y rápidamente progresiva. A nivel laríngeo los pacientes pueden referir disfonía por presencia de lesiones polipoídeas 0 por parálisis cordal ${ }^{4}$. El manejo generalmente es farmacológico con corticoides y/o inmunosupresores con buena respuesta a tratamiento médico ${ }^{8}$. Se han reportado casos de pacientes en los cuales posterior al manejo quirúrgico de la poliposis, han presentado en forma aguda síntomas característicos de vasculitis como púrpura, rash, neuropatías e hiperreosinofília 9 .

Se presenta paciente que consulta por rinosinusitus crónica poliposa y que por medio del hallazgo de características histológicas de SCS, se realiza el diagnóstico en un estadio precoz de su patología.

\section{CASO CLÍNICO}

Hombre, 64 años, consulta por obstrucción nasal de 15 años de evolución y otorrea bilateral ocasional. Al examen físico se evidencia otitis media crónica simple bilateral inactiva y poliposis nasosinusal bilateral grado 3 de Lildholdt. En los exámenes destaca audiometría con hipoacusia moderada bilateral con componente sensorioneural y conductivo; tomografía computarizada (TC) de cavidades paranasales con ocupación poliposa pansinusal, con dehiscencia de lámina papirácea izquierda con pólipo que protruye a la órbita con leve compresión de músculo recto medial (Figura 1).

Tabla 1. 1990 Academia Americana de Reumatología. Criterios diagnósticos para síndrome de Churg Straus

\begin{tabular}{|ll|}
\hline Criterio & Definición \\
\hline Asma & Historia de sibilancias o estertores difusos en espiración \\
Eosinofilia & Eosinofilos $>10 \%$ en muestra de sangre \\
Mono o polineuropatía & Mono o polineuropatía atribuible a vaculitis \\
Infiltrados pulmonares migratorios & Infiltrados pulmonares transitorios en radiografía \\
Anormalidades en senos paranasales & $\begin{array}{l}\text { Historia de molestias sinusales agudas o crónicas con compromiso } \\
\text { imaginológico }\end{array}$ \\
Eosinófilos extravasculares & Biopsia de arteria, arteriola o vena que muestre acumulación de eosinófilos en \\
& áreas extravasculares \\
\hline
\end{tabular}




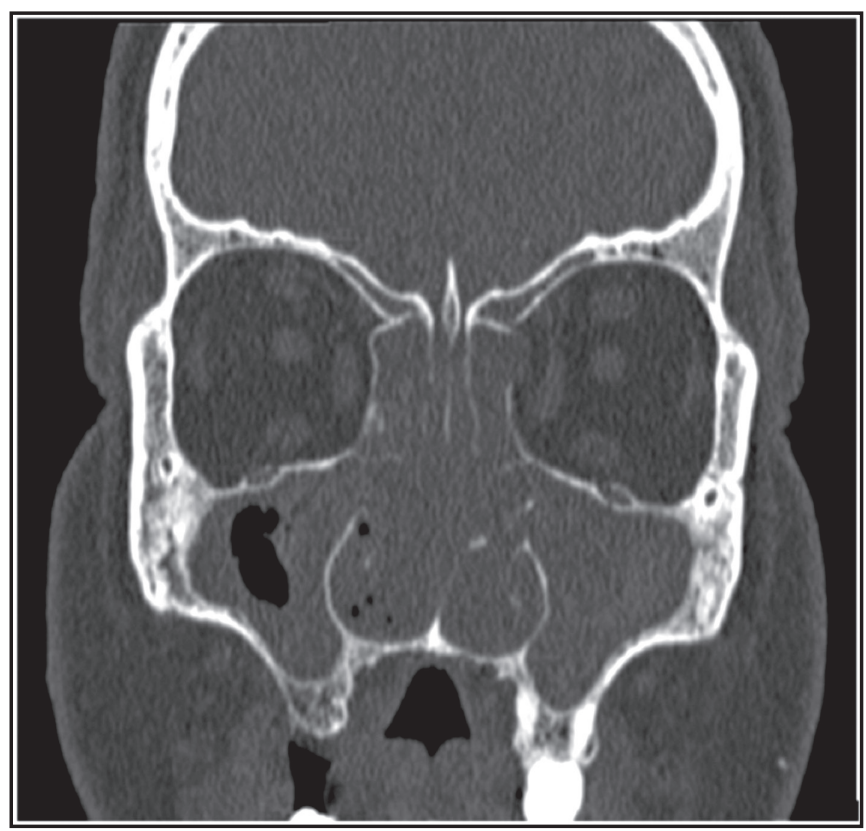

Figura 1. Tomografía computarizada de cavidades paranasales, corte coronal. Se observa poliposis nasosinusal severa con dehiscencia de la lámina papirácea izquierda.

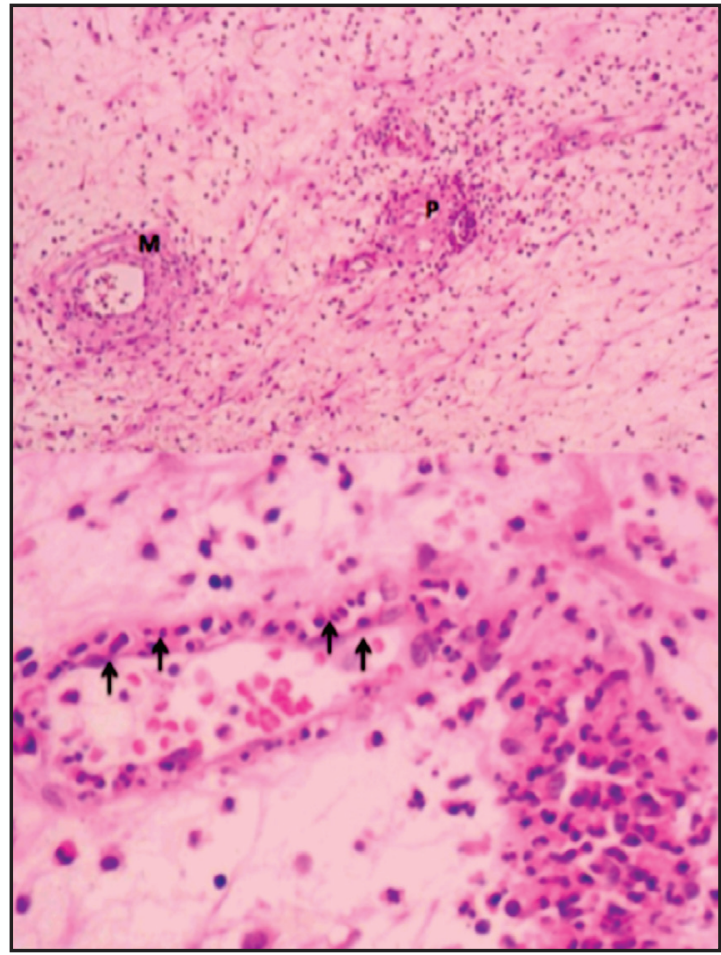

Figura 2. Biopsia de pólipos nasales: presencia de eosinófilos y polimorfonucleares de las paredes de vasos de mediano (M) y pequeño $(P)$ tamaño. Abajo las flechas indican infiltrado eosinofílico de pared de vaso de pequeño tamaño.
Dado edad tardía de presentación y severidad de poliposis se decide biopsiar lesiones en policlínico. La biopsia informa granulomatosis eosinofílica con poliangeítis (SCS) (Figura 2). Exámenes de laboratorio sin eosinofília, ANCA (-), función renal normal. Radiografía de tórax con infiltrado intersticial bibasal leve. Se envía a evaluación concomitante por equipos de inmunología, reumatología, broncopulmonar y nefrología; en esta evaluación solo destaca el hallazgo de limitación crónica del flujo aéreo. Luego de revisar la literatura y encontrar evidencia sobre reagudizaciones de la vasculitis posterior al manejo quirúrgico de la rinosinusitits crónica poliposa en estos pacientes ${ }^{9}$ y de buena respuesta a tratamiento farmacológico ${ }^{8}$, se decide no someter a cirugía rinosinusal, y se inicia manejo médico con corticoides orales, prednisona $10 \mathrm{mg}$ al día e inmunomoduladores, azatioprina $50 \mathrm{mg}$ al día, presentando franca disminución del tamaño de los pólipos nasales, grado 1 de Lidholdt. Por otra parte, paciente rechaza cirugía otológica, y se implementa con audífonos con buena respuesta. 


\section{CONCLUSIONES}

Es difícil encontrar pacientes con Churg Strauss en estadíos tempranos de la enfermedad. En el caso de este paciente consultó solo por síntomas otorrinolaringológicos y por medio del hallazgo de características histológicas de SCS se realizó el diagnóstico de su patología en un estadio precoz, otorgándole un tratamiento farmacológico oportuno, con buena respuesta clínica.

\section{BIBLIOGRAFÍA}

1. Castellano J et al. Sindrome de Churg Strauss. http://www.svreumatologia.com/wp-content/ uploads/2008/04/Cap-15-Sindrome-de-ChurgStrauss.pdf). [visita 10/05/2017].

2. Churg J, Strauss L. Allergic granulomatosis, allergic angiitis, and periarteritis nodosa. $A m \mathrm{~J}$ Pathol 1951; 27: 277-94.

3. Guillevin L et Al. Churg-Straus Syndrome: Clinical study and long term follow-up of 96 patients. Medicine 1999; 78: 26-37.

4. Olga E, Beltrán et al. Papel del otorrinolaringólogo en el diagnóstico y seguimiento de pacientes con vasculitis primarias. Reumatol Clin 2011; 7 Supl 3: 7-11 - Vol. 7.

5. Masi A et Al. The American College of Rheumato-
La similitud de los síntomas con la poliposis nasosinusal no asociada a vasculitis retarda el diagnóstico hasta verse lesiones agregadas a otro nivel. En base a este caso se podría inferir cierta utilidad en biopsiar los pólipos nasosinusales en el policlínico, al menos en pacientes con edad de presentación atípica, o rinosinusitis crónicas poliposas más severas, ya que de corresponder a esta patología, con un adecuado y precoz manejo médico se podría evitar la progresión de la enfermedad, la morbilidad quirúrgica y lograr disminuir los síntomas.

logy 1990 criteria for the classification of ChurgStrauss syndrome (allergic granulomatosis and angiitis). Arthritis Rheum 1990; 33: 1094-100.

6. Sinico RA et AL. Prevalence and clinical significance of antineutrophil cytoplasmatic antibodies in Churg Strauss syndrome. Arthritis Rheum 2005; 52: 2926-35.

7. Ginés David Parra-García et al. Manifestaciones otorrinolaringológicas de las vasculitis sistémicas. Acta Otorrinolaringol Esp 2012; 63: 303-10 - DOI: 10.1016/j.otorri.2011.09.002).

8. Bacciu A et Al. Nasal polyposis in Churg-Strauss syndrome. Laryngoscope 2008; 118: 325-9.

9. Fallah S et al. Churg Strauss Syndrome after Polypectomy in Asthmatic and Allergic Patients. Iran J Allergy Asthma Immunol 2009; 8: 111-15.

Dirección: Cecilia Sedano Muñoz 